\title{
Association of British Clinical Diabetologists (ABCD) Insulin Pump Network UK Committee position statement on the results of the REPOSE (Relative Effectiveness of Pumps Over Structured Education) trial
}

\author{
MARK EVANS'1 AND EMMA WILMOT² ON BEHALF OF THE ABCD INSULIN PUMP NETWORK UK \\ COMMITTEE
}

The results of the REPOSE (Relative Effectiveness of Pumps Over Structured Education) trial were published recently in the $B M J .{ }^{1}$ The $A B C D$ Insulin Pump Network Committee have become aware of a number of cases where the findings have been misreported and/or misinterpreted. We thought it would be helpful for readers of British Journal of Diabetes if we clarify the main REPOSE findings and implications for clinical practice in the UK.

In summary, the REPOSE study was a randomised controlled trial of insulin given by continuous subcutaneous insulin infusion (CSII) compared with insulin given by multiple daily injections (MDI), with both groups receiving structured education delivered through a one-week DAFNE (Dose Adjustment for Normal Eating) course. Participants were adults with type 1 diabetes (T1D) without a pressing and immediate clinical need for CSII (see below). Over the 24 months of the trial, improvements in glycaemic control (measured by $\mathrm{HbA}_{1 \mathrm{c}}$ and severe hypoglycaemia) and in measures of quality of life were seen in both arms of the study. The CSII group showed slightly greater improvements than the MDI arm, but most of the differences were not statistically significant, and the difference in $\mathrm{HbA}_{1 c}$ did not reach a clinically meaningful level. This has led some to interpret the study as showing that there is no benefit from CSII in T1D.

Currently, NICE technology appraisal guidance 151 (TA151)2 recommends CSII as a treatment option for adults and children aged 12 years and older with T1D, either where $\mathrm{HbA}_{1 \mathrm{c}}$ levels have remained high $(8.5 \%$ (69 $\mathrm{mmol} / \mathrm{mol})$ or above) on $\mathrm{MDI}$ therapy

Addenbrookes Hospital, Hills Road, Cambridge, UK

Derby Teaching Hospitals NHS Foundation Trust, Derby, UK

Address for correspondence: Dr Mark Evans

University Lecturer/Honorary Consultant Physician, Wellcome Trust/

MRC Institute of Metabolic Science, Institute of Metabolic Science

Metabolic Research Laboratories, University of Cambridge, Box 289

Addenbrookes Hospital, Hills Road, Cambridge CB2 OQQ, UK

Tel: +44 (0)1223 336994

E-mail: mle24@cam.ac.uk

Br J Diabetes 2017:17:73

http://dx.doi.org/10.15277/bjd.2017.132 despite a high level of care which would usually include structured education and/or where attempts to achieve target $\mathrm{HbA}_{1 \mathrm{c}}$ levels with MDIs result in the person experiencing disabling hypoglycaemia.

By design, the REPOSE study excluded patients who met the above TA151 criteria for CSII, examining whether CSII should be more widely available in T1D. Importantly, the results of REPOSE are thus not relevant to the patients currently covered by the evidence-based recommendations of TA151 and who would still be considered for CSII. REPOSE provided DAFNE education to both arms, finding sustained improvements in both arms in glycaemic control and measures of quality of life, reinforcing the use of structured education as an effective strategy in T1D.

In summary, the REPOSE findings support the current NICE T1D pathway, with structured education being offered first and CSII therapy then being considered where patients are still struggling to achieve glycaemic goals safely as set out in TA151. Sadly, most patients with T1D in the UK have not been offered evidence-based structured education. Similarly, access to CSII remains patchy, with the level of provision of CSII being lower than in other comparable countries. There is a danger that misrepresentation of the REPOSE data could worsen this inequality.

Conflict of interest MLE has received Advisory Board/speaker's fees and/or travel support from Medtronic, Roche, Cellnovo, Novonordisk, Eli Lilly and Abbott Diabetes Care.

EW has received personal fees from Medtronic and support to attend educational events from Cellnovo and Roche.

Funding None.

\section{References}

1. REPOSE Study Group. Relative effectiveness of insulin pump treatment over multiple daily injections and structured education during flexible intensive insulin treatment for type 1 diabetes: cluster randomised trial (REPOSE). BMJ 2017;356: j1285. http:dx.doi.org/10.1136/bmj.j1285

2. National Institute for Health and Care Excellence (NICE). Continuous subcutaneous insulin infusion for the treatment of diabetes mellitus. Guidance TA151. 2008. https://www.nice.org.uk/guidance/ta151 (accessed 18 May 2017). 